\title{
Visual object pattern separation varies in older adults
}

\author{
Heather M. Holden, ${ }^{1}$ Chelsea Toner, ${ }^{2}$ Eva Pirogovsky, ${ }^{3,4}$ C. Brock Kirwan, ${ }^{5,6}$ \\ and Paul E. Gilbert ${ }^{1,2,7}$
}

\begin{abstract}
${ }^{1}$ San Diego State University-University of California San Diego Joint Doctoral Program in Clinical Psychology, San Diego, California 92120, USA; ${ }^{2}$ Department of Psychology, ${ }^{3}$ Department of Psychiatry, San Diego State University, San Diego, California 92182, USA; ${ }^{4}$ Veterans Affairs, San Diego Health Care System, San Diego, California 92161, USA; ${ }^{5}$ Department of Psychology, ${ }^{6}$ Neuroscience Center, Brigham Young University, Provo, Utah 84602, USA
\end{abstract}

\begin{abstract}
Young and nondemented older adults completed a visual object continuous recognition memory task in which some stimuli (lures) were similar but not identical to previously presented objects. The lures were hypothesized to result in increased interference and increased pattern separation demand. To examine variability in object pattern separation deficits, older adults were divided into impaired and unimpaired groups based on performance on a standardized serial list-learning task. Impaired older adults showed intact recognition memory, but were impaired relative to young and unimpaired older adults when identifying similar lure stimuli, demonstrating that object pattern separation varies in older adults.
\end{abstract}

Pattern separation is defined as a mnemonic process responsible for separating partially overlapping patterns of activation so that one pattern may be retrieved as separate from other similar patterns. Theoretical models have hypothesized that pattern separation may be supported by the hippocampus (Marr 1971; McNaughton and Nadel 1989; O'Reilly and McClelland 1994; Rolls and Kesner 2006; Myers and Scharfman 2009). The operation of a pattern separation mechanism may be fundamental in reducing interference among similar memory representations, thus enhancing memory accuracy. There is considerable evidence that the dentate gyrus (DG) and CA3 hippocampal subregions play a critical role in pattern separation (for reviews, see Kesner 2007; Gilbert and Brushfield 2009; Rolls 2010; Yassa and Stark 2011; Schmidt et al. 2012).

The DG is particularly susceptible to age-related changes in animals (Small et al. 2004; Patrylo and Williamson 2007) and humans (Small et al. 2002). In addition, age-related changes are evident in the perforant pathway in humans (Yassa et al. 2011b) and animals (Geinisman et al. 1992). Wilson et al. (2006) suggest that age-related changes in the DG and diminished connections in the perforant path result in a reduced ability of the DG to resolve similarity among input patterns, resulting in less efficient pattern separation. In contrast, the recurrent collaterals of the CA3 subregion are hypothesized to become strengthened causing the CA3 auto-associative network to become entrenched in pattern completion. The result may be an age-related functional reorganization of hippocampal circuitry in which retrieval of previously stored information (pattern completion) becomes the dominant process at the expense of processing novel information (pattern separation). In support of this model, age-related changes in the integrity of the perforant pathway were associated with diminished pattern separation activity in the DG/CA3 subregions (Yassa et al. 2011b). These changes were suggested to weaken processing of novel information while strengthening processing of stored information. Changes in the cholinergic system also have been hypothesized to play a role in age-related cognitive dysfunction (for review, see Dumas and Newhouse 2011). Hasselmo and Wyble (1997) suggest that reduced acetylcholine to the hippo-

\footnotetext{
${ }^{7}$ Corresponding author

E-mail pgilbert.sdsu@gmail.com

Article is online at http://www.learnmem.org/cgi/doi/10.1101/Im.030171.112.
}

campus may drive the network into previously stored attractor states, while weakening the processing of new information in the CA3 network.

Given the critical role of the DG in supporting pattern separation and the susceptibility of this region to age-related changes, recent studies have begun to examine the effects of aging on the efficiency of the pattern separation mechanism in humans. Pattern separation has been shown to be less efficient in older adults on tasks involving spatial locations (Stark et al. 2010; Holden et al. 2012), visual objects (Toner et al. 2009; Yassa et al. 2011a; Stark et al. 2013), and temporal order of items in a sequence (Tolentino et al. 2012). Neuroimaging studies indicate that these age-related changes in pattern separation may be associated with functional and structural changes in the hippocampal network in older adults (Yassa et al. 2011a,b).

Although growing evidence indicates that pattern separation may be impaired in older adults, two recent studies suggest that there may be individual age-related variability in spatial pattern separation. Using different tasks, Stark et al. (2010) and Holden et al. (2012) found that when older adults were split into aged-impaired and aged-unimpaired groups based on standardized verbal learning test performance, the young adults and aged-unimpaired group performed significantly better than the aged-impaired group on trials hypothesized to tax pattern separation. However, very little is known about whether the differences reported in these studies are unique to spatial memory or whether the variability in pattern separation extends beyond the spatial domain. Only one study to our knowledge has investigated individual differences in pattern separation among older adults for visual object stimuli. Stark et al. (2013) found that pattern separation varied in older adults using an incidental encoding task. The purpose of the current study was to examine age-related variability in pattern separation efficiency for visual object information using a continuous recognition memory task that involved intentional encoding.

The present study used archival data from our laboratory that were included in a previous publication (Toner et al. 2009). The sample consisted of 20 nondemented older adults over $65 \mathrm{yr}(M=74.4 \mathrm{yr}$, SE 1.64$)$ and 20 young adults $(M=19.15 \mathrm{yr}$, SE 0.30). Older adults were screened for dementia using the Dementia Rating Scale (DRS; Mattis 1976). For the current study, the older adult group was split into two groups based on 
Table 1. Demographic data and DRS scores for young, older-unimpaired (OU) and older-impaired (OI) adults

\begin{tabular}{llllcc}
\hline & \multicolumn{1}{c}{ Young } & Unimpaired & \multicolumn{1}{c}{ Impaired } & Test statistic & $P$-value \\
\hline Age $(\mathrm{yr})$ & $19.15(0.302)$ & $72(1.88)$ & $76(2.39)$ & $F_{(1,18)}=1.46^{\mathrm{a}}$ & $P=0.24$ \\
DRS score & $\mathrm{N} / \mathrm{A}$ & $137.5(1.6)$ & $135.42(1.32)$ & $F_{(1,18)}=1.01^{\mathrm{a}}$ & $P=0.33$ \\
Education (yr) & $13.85(0.264)$ & $15.88(0.55)$ & $14.5(0.54)$ & $F_{(2,37)}=5.27$ & $P<0.05^{\mathrm{b}}$ \\
Gender (M/F) & $12 / 8$ & $3 / 5$ & $7 / 5$ & $\chi_{(1, N=40)}^{2}=1.25$ & $P=0.54$ \\
\hline
\end{tabular}

${ }^{a}$ One-way analysis of variance compared unimpaired vs. impaired older adults.

${ }^{b}$ Newman-Keuls post-hoc comparison test revealed older-unimpaired adults had significantly higher education level than young adults $(P<0.05)$. There were no other significant differences in years of education between groups.

performance on the Hopkins Verbal Learning Test-Revised (HVLT-R; Benedict et al. 1998; Brandt and Benedict 2001). As defined by Holden et al. (2012), older-unimpaired (OU) participants $(N=8)$ scored within the normal range for young adults (ages 20$29 \mathrm{yr}$ ) on the delayed recall subtest of the HVLT-R (mean words recalled 11, SD 1.07), while older-impaired (OI) participants $(N=$ 12) scored more than 1 standard deviation below these norms (mean words recalled 6.33, SD 1.5). It is important to note that the older-impaired adults were impaired relative to young adults on the HVLT-R but were not considered clinically impaired (defined as greater than 1.5 standard deviations below the norms for their age). HVLT-R scores were selected as the criterion to divide the older adults for several reasons. First, previous studies examining variability in pattern separation efficiency split older adults based on standardized verbal memory task performance (Stark et al. 2010, 2013; Holden et al. 2012). The utilization of a similar task in the present study allowed for more direct comparisons with prior studies. Second, given that pattern separation is a mnemonic process, it seemed most appropriate to split the group based on a test that assesses memory. Finally, serial list-learning tasks may be sensitive to medial temporal lobe function, which is implicated in pattern separation.

Demographic data for all groups and DRS scores for the older adult groups are provided in Table 1 . There were no significant differences between the OU and OI participants on demographic variables or DRS scores. Although the OI group was older, had less education, and had lower average DRS scores compared to the OU group, none of these variables were found to correlate significantly with the pattern separation task $(P \geq 0.10)$. In addition, analysis of variance revealed no significant differences between the $\mathrm{OU}$ and $\mathrm{OI}$ participants on a variety of neurocognitive tests, suggesting that general cognitive function did not differ significantly between the groups.

Participants completed a continuous recognition task developed by Kirwan and Stark (2007) during which they viewed pictures of everyday objects one at a time on a computer screen. Some objects were repeated across trials and some objects, labeled lures, were similar but not identical to objects previously presented. For each object, participants were asked to press a button to indicate whether the stimulus was: (1) new-the object had never been presented during the task; (2) old-the exact same object had been presented previously; or (3) similar-the object was similar, but not identical to one that had been presented previously during the task. Participants were administered six blocks of 108 stimuli. A given stimulus was repeated at most one time within a block and was never repeated across blocks.

The mean proportion of responses of new, old, and similar to first, repeated, and lure stimuli for the groups are displayed in Figure 1 . A $3 \times 3$ analysis of variance (ANOVA) with group (young, OU, OI) as the between-participant factor and stimulus (first, repeat, lure) as the within-participant factor was used to analyze correct responses. The analysis revealed a significant main effect of group, $F_{(2,37)}=16.30, \quad P<$ $0.001, \eta_{2}=0.43$; a significant main effect of stimulus, $F_{(2,74)}=98.81, P<0.001, \eta_{2}$ $=0.65$; and a group $\times$ stimulus interaction, $F_{(4,74)}=8.73, P<0.001, \eta_{2}=0.11$. A Newman-Keuls post-hoc comparison test of the interaction revealed that young and OU groups significantly outperformed the OI group in correctly identifying lures as similar. Effect sizes for group differences in the correct identification of lures were calculated using Cohen's $d$. Effect sizes were large for the comparison of young vs. OI $(d=2.31)$ and OU vs. OI $(d=1.561)$. There were no significant group differences $(P>0.05)$ in correctly identifying first stimuli as new or repeated stimuli as old.

To examine the types of responses to lure stimuli, difference scores were calculated for each participant by subtracting the number of "old" responses to lure stimuli (false positive) from the number of "similar" responses to lure stimuli (correct response). A one-way ANOVA with group (Young, OU, OI) as the between-participant factor and difference scores as the dependent variable revealed a significant group difference, $F_{(2,37)}=15.68$, $P<0.001$. A Newman-Keuls post-hoc comparison test revealed that young and OU were more likely to label a lure stimulus as similar, whereas OI were more likely to label a lure stimulus as old (Fig. 2). To further analyze types of errors committed by the OI group, a difference score was calculated subtracting the number of "new" responses to lure stimuli from the number of "old" responses to lures. A $t$-test with difference scores as the dependent variable revealed that OI participants mislabeled lure stimuli as old significantly more than new $t_{(11)}=5.92, P<0.001$.

These results reveal that young adults and OU significantly outperformed OI when correctly identifying lure stimuli as similar. Since there were no significant group differences in correctly identifying first stimuli as new or repeated stimuli as old, the group differences in correct responses to lures were not due solely to general recognition memory deficits. OI were more likely to commit false positive errors and label lure stimuli as old, providing evidence that pattern separation was less efficient in this group. These results suggest that deficits in visual object pattern separation may be variable in cognitively normal older adults. The current findings are consistent with three recent studies demonstrating individual differences in age-related pattern separation deficits for spatial (Stark et al. 2010; Holden et al. 2012) and visual object information (Stark et al. 2013). Inter-individual variability among aged individuals is evident in several aspects of memory, including recognition (Glisky et al. 1995; Christensen et al. 1999; Davidson and Glisky 2002; Wilson et al. 2002; Duarte et al. 2006) and immediate and delayed recall (Christensen et al. 1999; Wilson et al. 2002). However, the determinants of such variability are not well understood. Pattern separation is a mnemonic process that facilitates successful encoding, thus enhancing memory accuracy. The results of the current study, as well as of other recent studies demonstrating individual differences in pattern separation, suggest that efficiency of this mechanism may be a contributing factor in the variability in memory abilities in aged individuals.

Memory abilities have been linked to age-related neurobiological changes in the hippocampal formation. A recent longitudinal imaging study revealed that declines in episodic memory were associated with decreased hippocampal volume, as well as decreased activation in the left hippocampus, suggesting that structural and functional changes in the hippocampal formation are linked to memory decline (Persson et al. 2012). Small et al. 
(2002) reported that $60 \%$ of an older adult sample had diminished MRI signal in at least one hippocampal subregion and this hippocampal dysfunction was associated with declines in memory ability. In addition, the authors demonstrated that DG dysfunction is associated with normal aging, whereas signal decline in the entorhinal cortex (EC) is indicative of a pathological process. However, even in healthy adults, shrinkage of the EC is associated with poorer memory performance (Rodrigue and Raz 2004). In addition, age-related structural changes in the perforant path, as well as functional changes in the DG/CA3 regions, are correlated with diminished pattern separation activity (Yassa et al. 2011b). Yassa et al. (2011b) suggest that these age-related changes in the hippocampal network, and the corresponding pattern separation
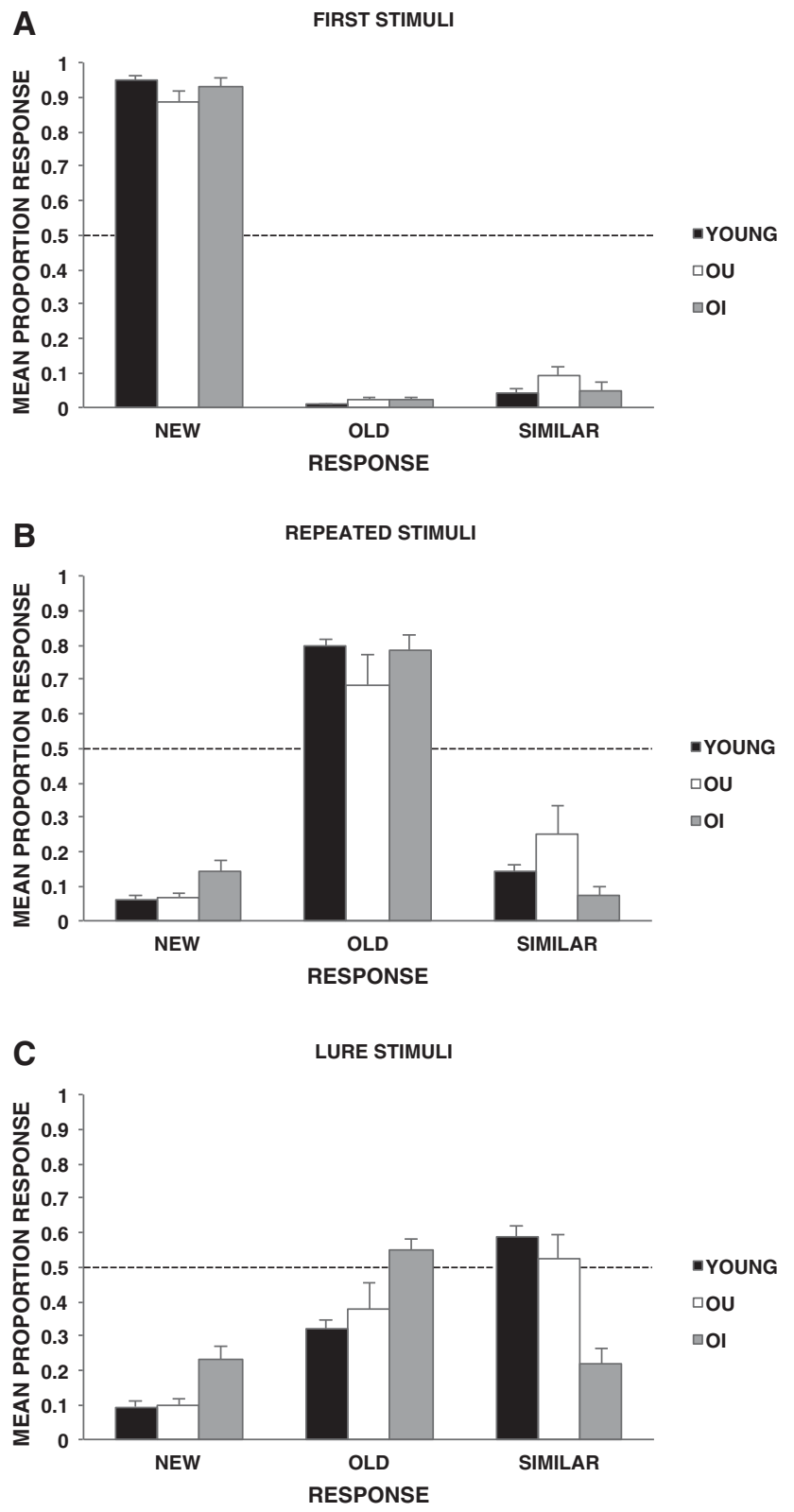

Figure 1. Mean proportion of responses $(+S E)$ of new, old, and similar to $(A)$ first presentation of objects (FIRST), $(B)$ repeated presentation of objects (REPEATED), and (C) objects that were similar but not identical to previously presented objects (LURE) for young, older-unimpaired $(\mathrm{OU})$, and older-impaired (OI) adults.

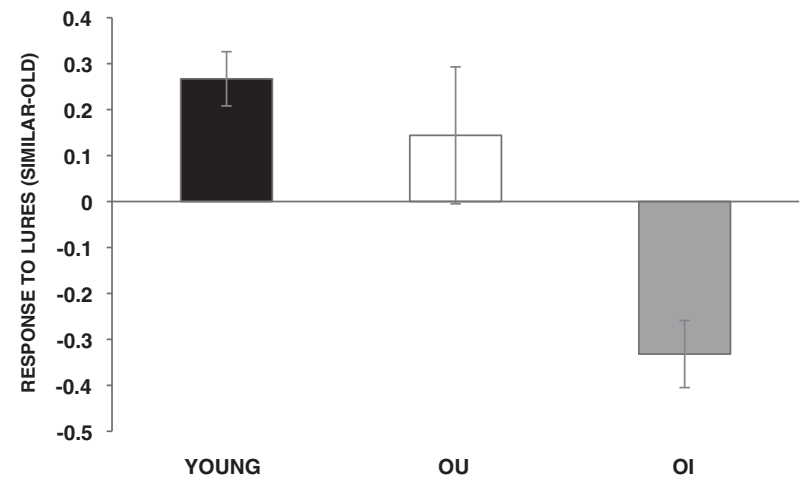

Figure 2. Mean difference scores: proportion of "similar" responses to lure stimuli (correct response)-proportion of "old" responses to lure stimuli (false positive response) for young, older-unimpaired (OU), and older-impaired $(\mathrm{OI})$ adults.

deficits, may lead to a shift from processing novel information to an over-reliance on retrieval of stored memory representations. The present finding that the OI group was more likely to identify lure stimuli as old rather than similar offers support for this hypothesis. The associations between these specific hippocampal subregions and age-related memory decline, in conjunction with the demonstrated role of these areas in supporting pattern separation, suggest that pattern separation efficiency may be an underlying mechanism subserving individual differences in memory decline in aging.

Although there is growing evidence to suggest that the human hippocampal subregions support pattern separation based on overlapping object features (Kirwan and Stark 2007; Bakker et al. 2008), there are data to suggest that perirhinal cortex also may play a role in pattern separation for visual objects. Lesion studies involving rats and monkeys indicate that lesions of the perirhinal cortex typically disrupt object recognition; however, hippocampal lesions produce variable results on object recognition tasks (Murray et al. 2000). Specifically, studies have shown that the perirhinal cortex may distinguish between visual objects with overlapping features to reduce feature ambiguity (Bussey et al. 2003, 2006; Gilbert and Kesner 2003; Norman and Eacott 2004; Bartko et al. 2007). However, there is some evidence to suggest that the hippocampus may play little role in disambiguating object features (Saksida et al. 2006). Aging studies have reported that total neuron numbers in rodents (Rapp et al. 2002) and cortical volumes in humans (Insausti et al. 1998) are largely preserved in the perirhinal cortex. However, researchers have postulated that age-related functional changes occur in perirhinal cortex in rodents (Moyer and Brown 2006) and humans (Ryan et al. 2012). Therefore, functional changes in the perirhinal cortex also may contribute to the decreased ability of older adults to identify lure stimuli as similar in the present study.

Future research should aim to identify the neurobiological underpinnings of individual differences in pattern separation in aging. It is important to identify whether there is corresponding inter-individual variability in dysfunction in specific hippocampal subregions and/or other regions such as the perirhinal cortex. Neurogenesis may play an important role in supporting mnemonic processes dependent on the DG, such as pattern separation (Clelland et al. 2009; Aimone et al. 2010; Deng et al. 2010, Sahay et al. 2011). Future investigations should examine potential associations between neurogenesis and individual differences in pattern separation abilities. In addition, evidence suggests that hippocampal subregions are differentially affected by normal and pathological aging processes (Braak and Braak 1996; West 
et al. 2000; Price et al. 2001; Small et al. 2002; Apostolova et al. 2010). It is unknown whether individual differences in pattern separation efficiency represent variability within the normal aging process or are indicative of early stages of a pathological process. If pattern separation deficits represent an early behavioral manifestation of future cognitive impairment, then tasks assessing pattern separation may be used in conjunction with standardized neuropsychological assessments to detect pathological changes. Finally, identification of older adults that perform similarly to young adults on these and other memory tasks provides a unique opportunity to further examine the factors involved in preserved memory function in aging. Future studies should aim to identify specific attributes of unimpaired older adults that may contribute to successful aging.

\section{Acknowledgments}

This research was supported by a National Institutes of Health Grant (\#AG034202) from the National Institute on Aging awarded to P.E.G. We thank Jerlyn Tolentino for assistance with participant recruitment and Shelley Jones, Peggy Ranke, Breeanna Bailey, and Adrienne Collazo for their assistance with data collection. We also thank all of the participants for their contributions to this study.

\section{References}

Aimone JB, Deng W, Gage FH. 2010. Adult neurogenesis: Integrating theories and separating functions. Trends Cogn Sci 14: 325-337.

Apostolova LG, Hwang KS, Andrawis JP, Green AE, Babakchanian S, Morra JH, Cummings JL, Toga AW, Trojanowski JQ, Shaw LM, et al. Alzheimer's Disease Neuroimaging Initiative. 2010. 3D PIB and CSF biomarker associations with hippocampal atrophy in ADNI subjects. Neurobiol Aging 31: 1284-1303.

Bakker A, Kirwan CB, Miller M, Stark CE. 2008. Pattern separation in the human hippocampal CA3 and dentate gyrus. Science 319: 1640-1642.

Bartko SJ, Winters BD, Cowell RA, Saksida LM, Bussey TJ. 2007. Perirhinal cortex resolves feature ambiguity in configural object recognition and perceptual oddity tasks. Learn Mem 14: 821-832.

Benedict RHB, Schretlen D, Groninger L, Brandt J. 1998. The Hopkins verbal learning test - revised: Normative data and analysis of inter-form and test-retest reliability. Clin Neuropsychol 12: 43-55.

Braak H, Braak E. 1996. Development of Alzheimer-related neurofibrillary changes in the neocortex inversely recapitulates cortical myelogenesis. Acta Neuropathol 92: 197-201.

Brandt J, Benedict RHB. 2001. Hopkins verbal learning test-revised. Professional manual. Psychological Assessment Resources, Lutz, FL.

Bussey TJ, Saksida LM, Murray EA. 2003. Impairments in visual discrimination after perirhinal cortex lesions: Testing 'declarative' vs. 'perceptual-mnemonic' views of perirhinal cortex function. Eur J Neurosci 17: 649-660.

Bussey TJ, Saksida LM, Murray EA. 2006. Perirhinal cortex and feature-ambiguous discriminations. Learn Mem 13: 103-105.

Christensen H, Mackinnon AJ, Korten AE, Jorm AF, Henderson AS, Jacomb P, Rodgers B. 1999. An analysis of diversity in the cognitive performance of elderly community dwellers: Individual differences in change scores as a function of age. Psychol Aging 14: 365-379.

Clelland CD, Choi M, Romberg C, Clemenson GD Jr, Fragniere A, Tyers P, Jessberger S, Saksida LM, Barker RA, Gage FH, et al. 2009. A functional role for adult hippocampal neurogenesis in spatial pattern separation. Science 325: 210-213.

Davidson PS, Glisky EL. 2002. Neuropsychological correlates of recollection and familiarity in normal aging. Cogn Affect Behav Neurosci 2: $174-186$.

Deng W, Aimone JB, Gage FH. 2010. New neurons and new memories: How does adult hippocampal neurogenesis affect learning and memory? Nat Rev Neurosci 11: 339-350.

Duarte A, Ranganath C, Trujillo C, Knight RT. 2006. Intact recollection memory in high-performing older adults: ERP and behavioral evidence. J Cogn Neurosci 18: 33-47.

Dumas JA, Newhouse PA. 2011. The cholinergic hypothesis of cognitive aging revisited again: Cholinergic functional compensation. Pharmacol Biochem Behav 99: 254-261.

Geinisman Y, deToledo-Morrell L, Morrell F, Persina IS, Rossi M. 1992. Age-related loss of axospinous synapses formed by two afferent systems in the rat dentate gyrus as revealed by the unbiased stereological dissector technique. Hippocampus 2: 437-444.
Gilbert PE, Brushfield AM. 2009. The role of the CA3 hippocampal subregion in spatial memory: A process oriented behavioral assessment. Prog Neuropsychopharmacol Biol Psychiatry 33: 774-781.

Gilbert PE, Kesner RP. 2003. Recognition memory for complex visual discriminations is affected by stimulus interference in rodents with perirhinal cortex damage. Learn Mem 10: 525-530.

Glisky EL, Polster MR, Routhieaux BC. 1995. Double dissociation between item and source memory. Neuropsychology 9: 229-235.

Hasselmo ME, Wyble BP. 1997. Free recall and recognition in a network model of the hippocampus: Simulating effects of scopolamine on human memory function. Behav Brain Res 89: 1-34.

Holden HM, Hoebel C, Loftis K, Gilbert PE. 2012. Spatial pattern separation in cognitively normal young and older adults. Hippocampus 22: $1826-1832$.

Insausti R, Juottonen K, Soininen H, Insausti AM, Partanen K, Vainio P, Laakso MP, Pitkänen A. 1998. MR volumetric analysis of the human entorhinal, perirhinal, and temporopolar cortices. AJNR Am J Neuroradiol 19: 659-671.

Kesner RP. 2007. A behavioral analysis of dentate gyrus function. Prog Brain Res 163: $567-576$

Kirwan CB, Stark CE. 2007. Overcoming interference: An fMRI investigation of pattern separation in the medial temporal lobe. Learn Mem 14: $625-633$.

Marr D. 1971. Simple memory: A theory for archicortex. Proc R Soc Lond B Biol Sci 262: 23-81.

Mattis S. 1976. Mental status examination for organic mental syndrome in the elderly patient. In Geriatric psychiatry: A handbook for psychiatrists and primary care physicians (ed. Bellack L, Katsau TB), pp. 77-121. Grune $\&$ Stratton, New York.

McNaughton BL, Nadel L. 1989. Hebbian-Marr networks and the neurobiological representation of action in space. In Neuroscience and connectionist theory (ed. Gluck MA, Rumelhart DE), pp. 1-63. Lawrence Erlbaum Associates, Hillsdale, NJ.

Moyer JR Jr, Brown TH. 2006. Impaired trace and contextual fear conditioning in aged rats. Behav Neurosci 120: 612-624.

Murray EA, Bussey TJ, Hampton RR, Saksida LM. 2000. The parahippocampal region and object identification. Ann N Y Acad Sci 911: $166-174$.

Myers CE, Scharfman HE. 2009. A role for hilar cells in pattern separation in the dentate gyrus: A computational approach. Hippocampus 19: $321-337$.

Norman G, Eacott MJ. 2004. Impaired object recognition with increasing levels of feature ambiguity in rats with perirhinal cortex lesions. Behav Brain Res 148: 79-91.

O’Reilly RC, McClelland JL. 1994. Hippocampal conjunctive encoding, storage, and recall: Avoiding a trade-off. Hippocampus 4: $661-682$.

Patrylo PR, Williamson A. 2007. The effects of aging on dentate circuitry and function. Prog Brain Res 163: 679-696.

Persson J, Pudas S, Lind J, Kauppi K, Nilsson LG, Nyberg L. 2012. Longitudinal structure-function correlates in elderly reveal MTL dysfunction with cognitive decline. Cereb Cortex 22: 2297-2304.

Price JL, Ko AI, Wade MJ, Tsou SK, McKeel DW, Morris JC. 2001. Neuron number in the entorhinal cortex and CA1 in preclinical Alzheimer disease. Arch Neurol 58: 1395-1402.

Rapp PR, Deroche PS, Mao Y, Burwell RD. 2002. Neuron number in the parahippocampal region is preserved in aged rats with spatial learning deficits. Cereb Cortex 12: 1171-1179.

Rodrigue KM, Raz N. 2004. Shrinkage of the entorhinal cortex over five years predicts memory performance in healthy adults. J Neurosci 24: $956-963$.

Rolls ET. 2010. A computational theory of episodic memory formation in the hippocampus. Behav Brain Res 215: 180-196.

Rolls ET, Kesner RP. 2006. A computational theory of hippocampal function, and empirical tests of the theory. Prog Neurobiol 79: 1-48.

Ryan L, Cardoza JA, Barense MD, Kawa KH, Wallentin-Flores J, Arnold WT, Alexander GE. 2012. Age-related impairment in a complex object discrimination task that engages perirhinal cortex. Hippocampus 22: 1978-1989.

Sahay A, Wilson DA, Hen R. 2011. Pattern separation: A common function for new neurons in hippocampus and olfactory bulb. Neuron 70: $582-588$.

Saksida LM, Bussey TJ, Buckmaster CA, Murray EA. 2006. No effect of hippocampal lesions on perirhinal cortex-dependent featureambiguous visual discriminations. Hippocampus 16: 421-430.

Schmidt B, Marrone DF, Markus EJ. 2012. Disambiguating the similar: The dentate gyrus and pattern separation. Behav Brain Res 226: 56-65.

Small SA, Tsai WY, DeLaPaz R, Mayeux R, Stern Y. 2002. Imaging hippocampal function across the human life span: Is memory decline normal or not? Ann Neurol 51: 290-295.

Small SA, Chawla MK, Buonocore M, Rapp PR, Barnes CA. 2004. Imaging correlates of brain function in monkeys and rats isolates a hippocampal 
subregion differentially vulnerable to aging. Proc Natl Acad Sci 101: 7181-7186.

Stark SM, Yassa MA, Stark CE. 2010. Individual differences in spatial pattern separation performance associated with healthy aging in humans. Learn Mem 17: 284-288.

Stark SM, Yassa MA, Lacy JW, Stark CE. 2013. A task to assess behavioral pattern separation (BPS) in humans: Data from healthy aging and mild cognitive impairment. Neuropsychologia doi: 10.1016 j.neuropsychologia.2012.12.014.

Tolentino JC, Pirogovsky E, Luu T, Toner CK, Gilbert PE. 2012. The effect of interference on temporal order memory for random and fixed sequences in nondemented older adults. Learn Mem 19: 251-255.

Toner CK, Pirogovsky E, Kirwan CB, Gilbert PE. 2009. Visual object pattern separation deficits in nondemented older adults. Learn Mem 16: $338-342$.

West MJ, Kawas CH, Martin LJ, Troncoso JC. 2000. The CA1 region of the human hippocampus is a hot spot in Alzheimer's disease. Ann N Y Acad Sci 908: $255-259$.
Wilson RS, Beckett LA, Barnes LL, Schneider JA, Bach J, Evans DA, Bennett DA. 2002. Individual differences in rates of change in cognitive abilities of older persons. Psychol Aging 17: 179-193.

Wilson IA, Gallagher M, Eichenbaum H, Tanila H. 2006. Neurocognitive aging: Prior memories hinder new hippocampal encoding. Trends Neurosci 29: 662-670.

Yassa MA, Stark CE. 2011. Pattern separation in the hippocampus. Trends Neurosci 34: 515-525.

Yassa MA, Lacy JW, Stark SM, Albert MS, Gallagher M, Stark CE. 2011a. Pattern separation deficits associated with increased hippocampal CA3 and dentate gyrus activity in nondemented older adults. Hippocampus 21: $968-979$.

Yassa MA, Mattfeld AT, Stark SM, Stark CE. 2011b. Age-related memory deficits linked to circuit-specific disruptions in the hippocampus. Proc Natl Acad Sci 108: 8873-8878.

Received December 21, 2012; accepted in revised form May 7, 2013. 


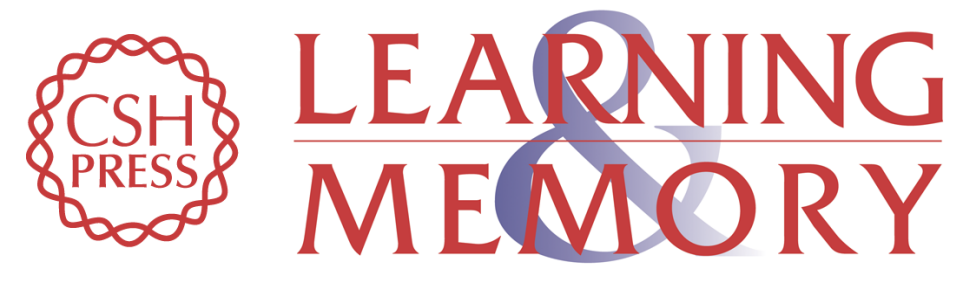

\section{Visual object pattern separation varies in older adults}

Heather M. Holden, Chelsea Toner, Eva Pirogovsky, et al.

Learn. Mem. 2013, 20:

Access the most recent version at doi:10.1101//m.030171.112

References This article cites 52 articles, 12 of which can be accessed free at: http://learnmem.cshlp.org/content/20/7/358.full.html\#ref-list-1

Creative This article is distributed exclusively by Cold Spring Harbor Laboratory Press for the Commons first 12 months after the full-issue publication date (see

License http://learnmem.cshlp.org/site/misc/terms.xhtml). After 12 months, it is available under a Creative Commons License (Attribution-NonCommercial 3.0 Unported), as described at http://creativecommons.org/licenses/by-nc/3.0/.

Email Alerting Receive free email alerts when new articles cite this article - sign up in the box at the Service top right corner of the article or click here. 